\title{
Room-temperature Sensing of Single Electrons using Vibrating-reed Electrometer in Silicon-on-Glass Technology
}

\author{
J. Jalil, Student Member, IEEE, Y. Ruan, and Y. Zhu, Senior Member, IEEE
}

\begin{abstract}
In the commonly-used existing silicon-on-insulator (SOI) microelectromechanical systems (MEMS) process, a siliconoxide-silicon sandwich structure in the sensor device creates parasitic capacitance, which reduces the charge sensitivity. In this letter, for the first time, we demonstrate a vibrating-reed based electrometry system with high sensitivity and resolution in the silicon-on-glass (SOG) MEMS process. In this work, we utilize a glass substrate instead of silicon to ameliorate charge sensitivity by reducing the parasitic capacitance. An improved preamplifier circuit topology incorporating with the proposed sensor shows a sensitivity of $1.43 \times 10^{11} \mathrm{~V} / \mathrm{C}$, which is the highest in the literature. In addition, the best charge resolution of $1.03 \mathrm{e} / \sqrt{ } \mathrm{Hz} @ 5.7 \mathrm{kHz}$ has achieved at room temperature and atmospheric pressure. This result provides strong evidence for the validity of our SOG based MEMS device as a charge sensor for detecting even a single electron.
\end{abstract}

Index Terms-Charge sensor, MEMS electrometer, resolution, sensitivity, silicon-on-glass (SOG), vibrating-reed (VR).

\section{INTRODUCTION}

The sensing of charge with high resolution plays an important role widening the use of electrometers or charge sensors in many diverse applications, such as surface charge analysis, mass spectrometry, tunneling microscopy, powder technology and aerosol science, bimolecular charge detection and ionization current measurement in ionization chambers [1]. The commercial transistor based electrometer, such as, the Keithley 6517A has a minimum charge resolution of $10 \mathrm{fC}(\sim$ $63 \mathrm{k}$ electrons) [2]. However, for bespoke scientific applications where charge noise of less than $1 \mathrm{fC}$ is required, Keithley 6517A's resolution is insufficient to meet the scientific needs. Recently, both resonator type and vibrating-reed (VR) type microelectromechanical systems (MEMS) electrometers exhibit significant improvement on charge sensing [1]. Nevertheless, the resonant MEMS sensors operated at room temperature suffer a poor charge resolution due to high phase noise. Thus, a vacuum environment is vital to achieve high quality factor ( $\mathrm{Q}$ factor) for overcoming phase noise issue to improve the device performance [3]-[8]. On the other hand, compared to the existing commercial macro-scale and resonator type MEMS charge sensors, the vibrating-reed based MEMS charge sensors exhibit superior charge resolution at room temperature, and vacuum environment is not required [9]-[13].

Until today, all micromachined charge sensing devices have been fabricated on silicon-on-insulator (SOI) substrates. Nonetheless, the existing SOI-MEMS vibrating-reed based electrometers undergo difficulty in achieving high sensitivity due to inherent parasitic capacitance $\left(C_{p a}\right)$, which is comparable to the time-modulated sense capacitance $\left(C_{v}\right)$ of few $\mathrm{pF}$ [9]-[13]. In this process, a single crystalline silicon (SCS) device layer lies on a buried oxide layer setting on top of a silicon substrate. Therefore, in SOI-MEMS devices, a major contributor to the parasitic capacitance is the static

J. Jalil and Y. Zhu are with Queensland Micro and Nanotechnology Centre, Griffith University, Nathan, QLD 4111, Australia. (e-mail: y.zhu@griffith.edu.au).

Y. Ruan is with Department of Precision Instruments, Tsinghua University, Beijing, People's Republic of China. (e-mail: ruanyong@mail.tsinghua.edu.cn).

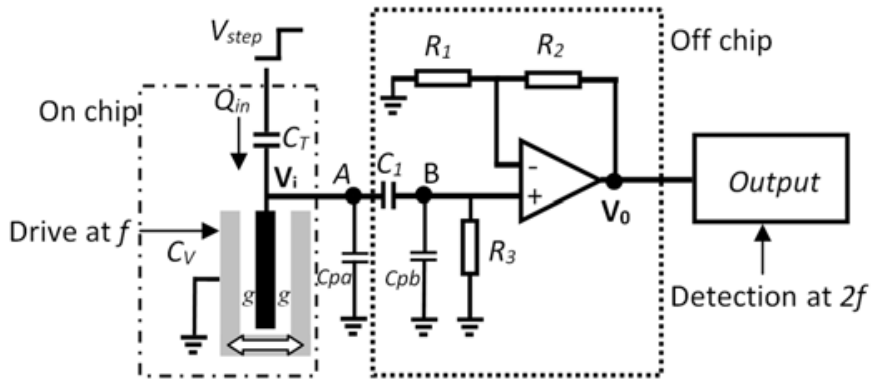

Fig. 1. Schematic of preamplifier and MEMS VR charge sensor.

capacitance between the sense electrodes and grounded substrate. Even though the underlying substrate of the device is removed, the parasitic capacitance still exists in the pad area because of siliconoxide-silicon sandwich structures. To overcome the inherent issue of the static parasitic capacitance between the pad and grounded substrate in SOI-MEMS, we propose in this letter a unique siliconon-glass (SOG) based vibrating-reed charge sensing system. Here, a silicon/glass anodic bonding technology [14]-[15] has been exploited, and the use of glass wafer as a substrate instead of silicon can make the parasitic capacitance minimal for not forming any siliconoxide-silicon structures. Hence, the sensor's sensitivity has been enhanced significantly in comparison with SOI counterparts. Both charge sensitivity and intrinsic noise level of the readout circuit determine the minimum charge detection of an electrometry system. Hence, a low noise and low leakage opamp based preamplifier has been designed to achieve high charge resolution.

\section{DESIGN AND FABRICATION}

Vibrating-reed charge sensors use a charge modulation technique that increases the operating frequency $(f)$ of MEMS sensors so that it can avoid flicker noise effect [9], [16]. In this technique, the input charge $\left(Q_{i n}\right)$ to be measured is loaded on the sense electrodes and upmodulated to an AC voltage signal, whose frequency is twice of the operating frequency and well above the noise corner frequency of the chosen readout electronics. Fig. 1 shows a schematic of the designed voltage-mode preamplifier and the SOG-MEMS sensing device along with on-chip test capacitance $\left(C_{T}\right)$. Before being processed, the $\mathrm{AC}$ sensing signal $\left(V_{i}\right)$ of SOG MEMS charge sensor is conditioned by a passive high-pass filter and a non-inverting operational amplifier. Both MOSFET and JFET based opamps can be implemented to design the preamplifier. However, in comparison with MOSFETs, JFET based opamps exhibit low input voltage noise and low input capacitance. Therefore, we use a low noise JFET input based opamp (AD8067) having $1 \mathrm{~T} \Omega$ input resistance and $2.5 \mathrm{pF}$ input capacitance to design the preamplifier circuit [17]. A gain resistor $\left(R_{1}=680 \Omega\right)$ and a feedback resistor $\left(R_{2}=47 \Omega\right)$ are chosen for a DC voltage gain of $15.47 \mathrm{~V} / \mathrm{V}$.

In the context of a preamplifier of charge sensor, the leakage current refers to a tiny DC current $(\sim \mathrm{pA})$ presented in the opamp's input terminals. In high-precision electrometry, this tiny leakage current is much larger than the input charge to be measured. To 


\section{Glass}

(a) Metallization

\section{Silicon}

\section{(b) Shallow trench etching}

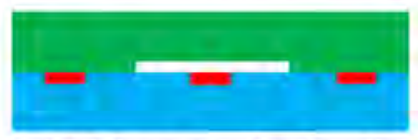

(c) Si/glass anodic bonding
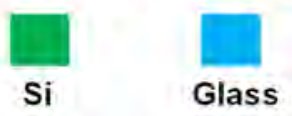

TABLE |

DEVICE PARAMETERS

Structure thickness $(\mu \mathrm{m})$

Suspension beam width $(\mu \mathrm{m})$

Suspension beam length $(\mu \mathrm{m})$

Sense capacitor gap, $g(\mu \mathrm{m})$

Total spring constant $(\mathrm{N} / \mathrm{m})$

umber of sense combs per side

Sense capacitance $(\mathrm{pF})$

\begin{tabular}{|c|}
\hline Value \\
\hline 32 \\
3.8 \\
404 \\
2.85 \\
5.6 \\
33 \\
144 \\
2.49 \\
\hline
\end{tabular}

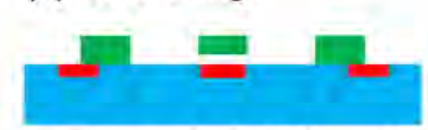

(f) Removal of photoresist

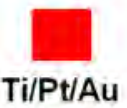

\section{Photoresist}

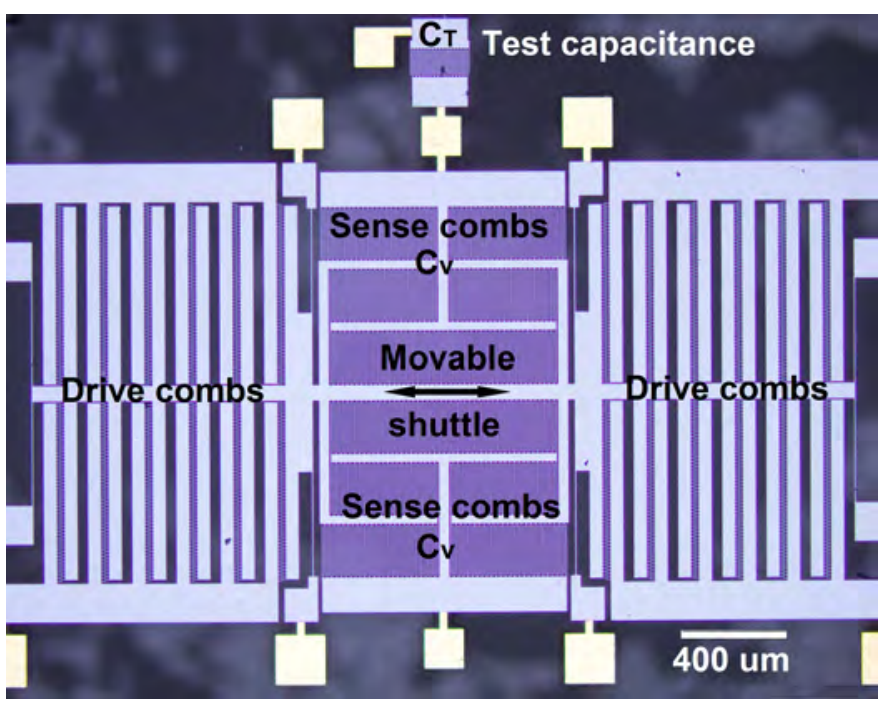

Fig. 3. Microphotograph of SOG-MEMS VR charge sensing device.

approximate $32 \mu \mathrm{m}$ by $\mathrm{KOH}$ etching (Fig. 2d). Following, the sensor structure is defined in the photoresist by photolithography, and etched using high aspect ratio ICP etching (Fig. 2e). Finally, the photoresist is removed from the device using oxygen plasma etching (Fig. 2f). The final fabricated SOG-MEMS device is shown in Fig. 3.

\section{Results And Discussion}

The fabricated MEMS device is wire-bonded in an open cavity ceramic package, and tested directly on a customized PCB comprising both driver and preamplifier circuits. As shown in Fig. 3, the push-pull comb drive actuators are placed at both ends of the moving shuttle, while the parallel plate electrodes that form the sensing capacitor $\left(C_{v}\right)$ are located in the middle. To maximize the capacitance variation at a given excitation voltage, actuation is carried out at the first inplane natural frequency $(f)$ of the MEMS structure. Known quantities of charge $\left(Q_{i n}\right)$ are introduced on the anchored sense electrodes by adding known step voltages to a $274 \mathrm{fF}$ on-chip test capacitor $\left(C_{T}\right)$. Increasing levels of step voltages $\left(V_{\text {step }}\right)$ are applied to the test capacitor using a voltage source. In steps of $0.1 \mathrm{~V}$, the test capacitor calibrates the amount of charge on the electrometer input, and the measured output is monitored on a digital oscilloscope in fast Fourier transform (FFT) mode. All measurements are conducted at room temperature and ambient pressure.

Initially, a sinusoidal voltage of $5 \mathrm{Vpp}$ along with a DC bias voltage of $40 \mathrm{~V}$ is applied at $2.85 \mathrm{kHz}$ to drive the device. The detection is carried out at $5.7 \mathrm{kHz}$, which is well above the flicker noise corner frequency $(2 \mathrm{kHz})$, and well below the maximum bandwidth (54 $\mathrm{MHz})$ of AD8067 operational amplifier. The displacement ratio $(x / g)$ of the resonator was found to be $1 / 2$ under a microscope. Fig. 4 shows MEMS electrometer's response with respect to input charge. The input-to-output relationship is highly linear, and the slope of the best-fit line is $3.65 \times 10^{11} \mathrm{~V} / \mathrm{C}$, which is the output voltage to input charge conversion gain. The overall preamplifier system voltage gain is measured to be $5.6 \mathrm{~V} / \mathrm{V}$ at $5.7 \mathrm{kHz}$, while input signal is applied at the node A in Fig. 1. Therefore, the sensor's sensitivity is $6.51 \times 10^{10} \mathrm{~V} / \mathrm{C}$ without considering the voltage gain of preamplifier. The displacement ratio $(x / g)$ plays a pivotal role in obtaining high charge sensitivity and needs to be as large as possible [9]-[13]. To achieve larger displacement amplitude $(x)$ of the shuttle, the moving combs are actuated by applying a higher DC bias voltage of $46 \mathrm{~V}$ and $\mathrm{AC}$ voltage of $7.5 \mathrm{Vpp}$ at $2.85 \mathrm{kHz}$. The displacement amplitude 


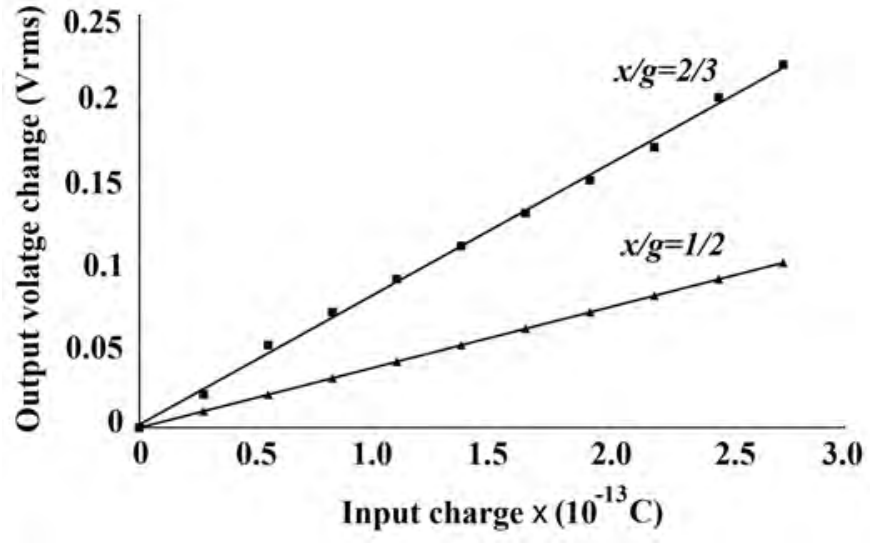

Fig. 4. Measured MEMS electrometer output voltage change versus input charge.

of the resonator is found to be $2 / 3$ of the sense capacitor's gap $(g)$ Thus, a higher sensitivity of $1.43 \times 10^{11} \mathrm{~V} / \mathrm{C}$ is achieved with $2 / 3$ displacement ratio.

The main noise sources in the proposed preamplifier are opamp input-referred voltage noise $\left(V_{n}\right)$ and current noise $\left(I_{n}\right)$, and thermal noises of the resistors. Input noise voltage and noise current of AD8067 are $7.5 \mathrm{nV} / \sqrt{ } \mathrm{Hz}$ and $0.6 \mathrm{fA} / \sqrt{ } \mathrm{Hz}$ respectively at $5.7 \mathrm{kHz}$. An optimized value of $50 \mathrm{G} \Omega$ for $R_{3}$ is selected to improve noise performance and ensure the output of the opamp not being saturated. The output noise voltage spectral density of the proposed circuit is $132.3 \mathrm{nV} / \sqrt{ } \mathrm{Hz}$ at $5.7 \mathrm{kHz}$. Charge resolution can be derived by dividing the output voltage noise density by the output voltage to input charge conversion gain at the frequency of interest. Thus, the resolution of the device is determined to be $0.165 \mathrm{aC} / \sqrt{ } \mathrm{Hz}$, which is equivalent to $1.03 \mathrm{e} / \sqrt{ } \mathrm{Hz}$ at $5.7 \mathrm{kHz}$.

As shown in Table 2, the proposed electrometer demonstrates excellent sensitivity and resolution compared to all SOI-MEMS based electrometers including both vibrating-reed and resonator types as well as contemporary commercial electrometers, such as Keithley 6517A [2] and Keysight B2987A [18]. The SOI-MEMS charge sensor of [10] is one of authors' previous work, and the design layout and parameters are kept the same with the sensor in this letter. Due to the reduction of parasitic capacitance of the device, the SOGMEMS electrometer shows 2.4 times higher sensitivity compared to the SOI counterpart with the same displacement ratio of $1 / 2$. The vibrating capacitance of the sensor is $2.49 \mathrm{pF}$, which is higher than the equivalent series capacitance of $C_{1}$ and $C_{p b}$. Thus, a large amount of input charge accumulates onto the vibrating capacitor. On the contrary, the input charge in [10] and [13] was distributed largely on circuit parasitic capacitances, and thereby affects the performance of sensitivity. Moreover, our proposed design shows wider dynamic range of sensitivity in contrast to [10] and [12]. Regarding charge resolution, the proposed sensor outperforms the recently published MEMS resonator type charge sensor exhibiting resolution of 9.21 $e / \sqrt{ } \mathrm{Hz}[8]$.

\section{CONCLUSION}

A new MEMS vibrating-reed electrometry system in SOG process has been designed, fabricated and characterized. The response is shown to be linear within the range of input charge tested at room temperature and atmospheric pressure. By selecting a lower voltage noise opamp, the further reduction of the noise floor of the readout circuit could be possible for improving charge resolution to subelectrons. The high charge sensitivity of SOG-MEMS electrometer
TABLE ॥

COMPARISON OF MEMS AND COMMERCIAL ELECTROMETERS AT ROOM TEMPERATURE

\begin{tabular}{|cccccl|}
\hline Year [Ref] & Sensor type & Wafer & Pressure & Sensitivity & $\begin{array}{l}\text { Resolution } \\
(e / \sqrt{ } \mathrm{Hz})\end{array}$ \\
\hline $2004[2]$ & Transistor & - & Ambient & - & $63 \mathrm{k}$ \\
$2014[18]$ & Transistor & - & Ambient & - & $6.3 \mathrm{k}$ \\
$2017[6]$ & Resonator & SOI & $33 \mathrm{mTorr}$ & $4.4 \times 10^{-4} \mathrm{~Hz} / f C^{2}$ & $203 \mathrm{k}$ \\
$2015[4]$ & Resonator & SOI & $40 \mathrm{mTorr}$ & $1.3 \times 10^{-3} \mathrm{~Hz} / f C^{2}$ & $131 \mathrm{k}$ \\
$2008[3]$ & Resonator & SOI & 4 mTorr & $1.2 \times 10^{-3} \mathrm{~Hz} / f C^{2}$ & $25 \mathrm{k}$ \\
$2018[7]$ & Resonator & SOI & Vacuum & $7.86 \times 10^{-3} \mathrm{~Hz} / f C^{2}$ & $16 \mathrm{k}$ \\
$2016[5]$ & Resonator & SOI & 20 mTorr & $6.3 \times 10^{-4} \mathrm{~Hz} / f C^{2}$ & $7.9 \mathrm{k}$ \\
$2018[8]$ & Resonator & SOI & Vacuum & $22.7 \times 10^{-3} / f C$ & 9.21 \\
$2008[12]$ & VR & SOI & Ambient & $1.28 \times 10^{10} \mathrm{~V} / \mathrm{C}$ & 52.4 \\
$2013[13]$ & VR & SOI & Ambient & $2.7 \times 10^{9} \mathrm{~V} / \mathrm{C}$ & 23 \\
$2008[10]$ & VR & SOI & Ambient & $2.74 \times 10^{10} \mathrm{~V} / \mathrm{C}$ & 6 \\
This work $*$ & VR & SOG & Ambient & $6.51 \times 10^{10} \mathrm{~V} / \mathrm{C}$ & 2.36 \\
This work $\dagger$ & VR & SOG & Ambient & $1.43 \times 10^{11} \mathrm{~V} / \mathrm{C}$ & 1.03 \\
\hline
\end{tabular}

$*$ and $\dagger$ denote displacement ratio of the SOG-MEMS vibrating-reed sensor of $1 / 2$ and $2 / 3$, respectively.

shows the potential in low-cost, portable instrumentation systems. The temperature sensitivity, long-term output drift and system integration of the sensor will be investigated in our future work.

\section{REFERENCES}

[1] J. Jalil, Y. Zhu, C. Ekanayake, and Y. Ruan, "Sensing of single electrons using micro and nano technologies: a review," Nanotechnology, vol. 28, no. 14, p. 142002, Mar. 2017, https://doi.org/10.1088/1361-6528/aa57aa.

[2] Low level measurements handbook, Keithley Instruments Inc., Cleveland, Ohio, USA, 2004 [Online]. Available: http://web.mit.edu/8.13/8.13d/manuals/LowLevMsHandbk.pdf, Accessed on Feb. 21, 2016.

[3] J. E. Y. Lee, B. Bahreyni, and A. A. Seshia, "An axial strain modulated double-ended tuning fork electrometer," Sens. Actuator A-Phys., vol. 148, no. 2, pp. 395-400, Dec. 2008, https://doi.org/10.1016/j.sna.2008.09.010.

[4] J. Zhao, H. Ding, and J. Xie, "Electrostatic charge sensor based on a micromachined resonator with dual microlevers," Appl. Phys. Lett., vol. 106, no. 23, p. 233505, Jun. 2015, https://doi.org/10.1063/1.4922458.

[5] H. Zhang, W. Yuan, J. Huang, B. Li, and H. Chang, "A high-sensitivity micromechanical electrometer based on mode localization of two degreeof-freedom weakly coupled resonators," J. Microelectromech. Syst., vol. 25, no. 5, pp. 937-946, Oct. 2016, DOI:10.1109/JMEMS.2016.2598780.

[6] D. Chen, J. Zhao, Y. Wang, Z. Xu, and J. Xie, "An electrostatic charge sensor based on micro resonator with sensing scheme of effective stiffness perturbation," J. Micromech. Microeng., vol. 27, no. 6, p. 065002, Apr. 2017, https://doi.org/10.1088/1361-6439/aa6b41.

[7] D. Chen, J. Zhao, Y. Wang, Z. Xu, and J. Xie, "Sensitivity manipulation on micro-machined resonant electrometer toward high resolution and large dynamic range," Appl. Phys. Lett., vol. 112, no. 1, p. 013502, Jan. 2018, https://doi.org/10.1063/1.5009276.

[8] J. Yang, H. Kang, and H. Chang, "A micro resonant electrometer with 9-electron charge resolution in room temperature," in Proc. IEEE Micro Electro Mechanical Systems (MEMS), Belfast, Jan. 2018, pp. 67-70, DOI: 10.1109/MEMSYS.2018.8346484.

[9] P. S. Riehl, K. L. Scott, R. S. Muller, R. T. Howe, and J. A. Yasaitis, "Electrostatic charge and field sensors based on micromechanical resonators," J. Microelectromech. Syst., vol. 12, no. 5, pp. 577-589, Oct. 2003, DOI: $10.1109 /$ JMEMS.2003.818066.

[10] J. Lee., Y. Zhu, and A. Seshia, "Room temperature electrometry with SUB-10 electron charge resolution," J. Micromech. Microeng., vol. 18, no. 2, p. 025033, Jan. 2008, https://doi.org/10.1088/09601317/18/2/025033.

[11] Y. Zhu, J. Lee, and A. Seshia, "System-level simulation of a micromachined electrometer using a time-domain variable capacitor circuit model," J. Micromech. Microeng., vol. 17, no. 5, pp. 1059-1065, Apr. 2007, https://doi.org/10.1088/0960-1317/17/5/028.

[12] Y. Zhu, J. E. Y. Lee, and A. A. Seshia, "A resonant micromachined electrostatic sensor," IEEE Sensors J., vol. 8, no. 9, pp. 1499-1505, Jul. 2008, DOI: 10.1109/JSEN.2008.923597. 
[13] G. Jaramillo, C. Buffa, M. Li, F. J. Brechtel, G. Langfelder, and D. A. Horsley, "MEMS electrometer with femtoampere resolution for aerosol particulate measurements," IEEE Sensors J., vol. 13, no. 8, pp. 29933000, Jun. 2013, DOI: 10.1109/JSEN.2013.2266335.

[14] J. He, X. Huang, L. Zhang, D. Zhao, F. Yang, W. Wang, and D. Zhang, "Mechanical strength of anchormicrobeam combined structure fabricated by silicon-on-glass process," IET Micro Nano Lett., vol. 9, no. 10, pp. 660-663, Aug. 2014, DOI:10.1049/mnl.2014.0251.

[15] J. Wang, Z. Yang, and G. Yan, "A silicon-on-glass Z-axis accelerometer with vertical sensing comb capacitors," in Proc. IEEE 7th Int. Conf. Nano/Micro Engineered and Molecular Syst. (NEMS), Kyoto, May 2012, pp. 583-586, DOI: 10.1109/MEMSYS.2018.8346484.

[16] H. Paleosky, R. K. Swank, and R. Grenchik, "Design of dynamic condenser electrometers," Rev. Sci. Instrum., vol. 18, no. 5, pp. 298314, May 1947, https://doi.org/10.1063/1.1740941.

[17] Analog Devices, Datasheet AD8067 [Online]. Available: http://www.analog.com/media/en/technical-documentation/datasheets/AD8067.pdf, Accessed on: Apr. 1, 2017.

[18] Keysight B2980 Series Femto/Picoammeter Electrometer/High Resistance Meter, Keysight Technologies [Online]. Available: https://literature.cdn.keysight.com/litweb/pdf/B2980-90010.pdf, Accessed on Sep. 28, 2018. 